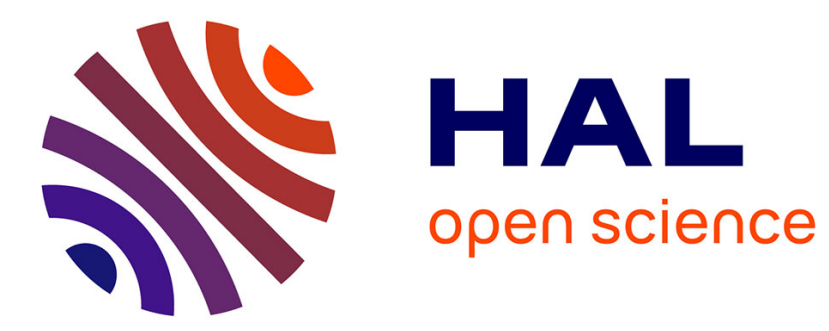

\title{
Photothermally modulated playback from a magnetic tape
}

\author{
J. Pelzl, M. Kaack, Th. Orth, K. Friedrich
}

\section{To cite this version:}

J. Pelzl, M. Kaack, Th. Orth, K. Friedrich. Photothermally modulated playback from a magnetic tape. Journal de Physique IV Proceedings, 1994, 04 (C7), pp.C7-3-C7-6. 10.1051/jp4:1994701 . jpa-00253142

\section{HAL Id: jpa-00253142 https://hal.science/jpa-00253142}

Submitted on 1 Jan 1994

HAL is a multi-disciplinary open access archive for the deposit and dissemination of scientific research documents, whether they are published or not. The documents may come from teaching and research institutions in France or abroad, or from public or private research centers.
L'archive ouverte pluridisciplinaire HAL, est destinée au dépôt et à la diffusion de documents scientifiques de niveau recherche, publiés ou non, émanant des établissements d'enseignement et de recherche français ou étrangers, des laboratoires publics ou privés. 


\title{
Photothermally modulated playback from a magnetic tape
}

J. Pelzl, M. Kaack, Th. Orth and K. Friedrich(1)

Institut für Experimentalphysik III, Festkörperspektroskopie, Ruhr-Universität, 44780 Bochum, Germany

\begin{abstract}
A novel method is presented which allows spatially resolved detection of magnetic signals from tapes. The PM-playback signal is generated in the head of a tape recorder by thermal modulation of the magnetization of the stationary tape. The PM-playback has been observed from homogeneously magnetized $\mathrm{Fe}_{2} \mathrm{O}_{3}, \mathrm{CrO}_{2}$ and ferrochrome tapes and from a sinusoidal track recorded on a $\mathrm{CrO}_{2}$ tape. From the signals measured as a function of laser spot position and of modulation frequency the depth and lateral variation of the magnetisation has been determined.
\end{abstract}

\section{INTRODUCTION}

Photothermal methods which offer a spatially resolved information of magnetization distributions so far rely on the microwave resonance absorption of the magnetic moments [1]. Applying the photothermally modulated ferromagnetic resonance, it has been possible to visualise signal traces recorded on magnetic tapes [2,3]. However, this technique has two main draw-backs. The ferromagnetic resonance experiment requires a complex and expensive technical arrangement which cannot be easily transferred to an industrial application. Secondly, to drive the magnetic material through the resonance condition an external magnetic field is needed which can perturb or even destroy the track of the magnetic recording. Here we present a new method, the photothermally modulated (PM) playback, that avoids these difficulties. The PM-playback is also based on the local generation of thermal waves in the magnetic recording media and therefore provides a lateral and depth resolved detection of the magnetization distribution of a magnetic tape. The principle of this method is sketched in Fig. 1.

The regular playback-process relies on the induction of a voltage in the head coils by a moving tape. In the PM-playback-method the tape is fixed on the magnetic head and the time dependent magnetic flux is obtained by the thermal modulation of the magnetization of the stationary tape. For this purpose the magnetic tape that is in contact with the head is illuminated by an intensity modulated focused laser beam 


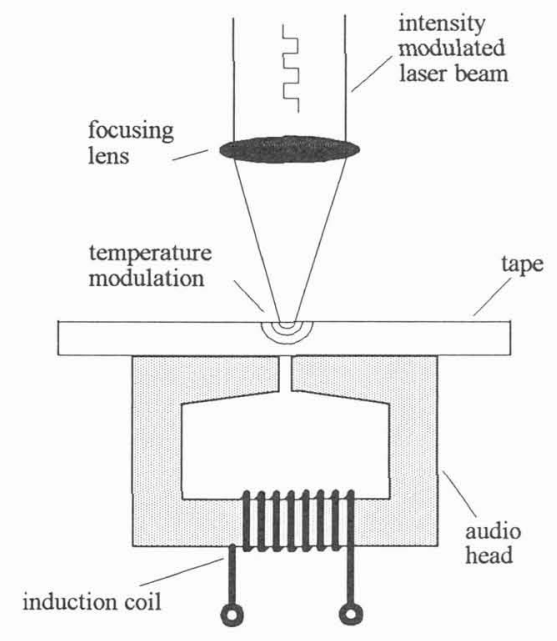

Fig. 1. Schematic of the photothermally modulated playback method for spatially resolved detection of magnetisation distribution on a magnetic tape.

(1). The induced read voltage that arise from a photothermal modulation of the magnetic flux around the head gap is then governed by the temperature derivative of the magnetization [5].

$$
U=\varepsilon \cdot \frac{d}{d t} \iiint \vec{M} \cdot \vec{H} d V=2 i \omega N W \hat{H} \varepsilon \cdot \int \frac{d M(x)}{d T} \Theta(x) d x
$$

By the integral in the right expression of (1) the temperature derivative of the magnetization of the tape is weighted with the variation of ac-temperature amplitude $\Theta(x)$ perpendicular to the tape plane, providing depth sensitive information. Due to the factor $\omega$ in the above relation, the signal amplitude does not decrease at high modulation frequencies $\omega$ although the ac-temperature amplitude decreases. $\Theta(x)$ is determined in the frame of a one-dimensional heat diffusion problem, adopting a trilayer composed of an air gap between the head and the magnetic layer which is supported by a polyester backing.

\section{EXPERIMENTAL AND RESULTS}

The experimental set-up used in this work consists of an audio-stereo head followed by a broad band amplifier. The local thermal modulation is achieved by a He-Ne laser focused to spot of about $20 \mu \mathrm{m}$ diameter on the tape. On one track of the stereo-tape a $50 \mathrm{~Hz}$ sine-signal had been recorded with and without acbiasing. The tape was fixed on the head, which was mounted on a translation stage to allow the lateral movement of the laser spot across the tape surface. The output voltage of the audio head was recorded by a lock-in amplifier synchronously with the intensity modulation of the heating light beam. The amplitude and the phase of the signal was measured as a function of the modulation frequency in the range $10 \mathrm{~Hz}$ to $100 \mathrm{kHz}$ and as a function of the laser spot on the tape. The signals were normalised with respect to the signal of the unmagnetized tape. 


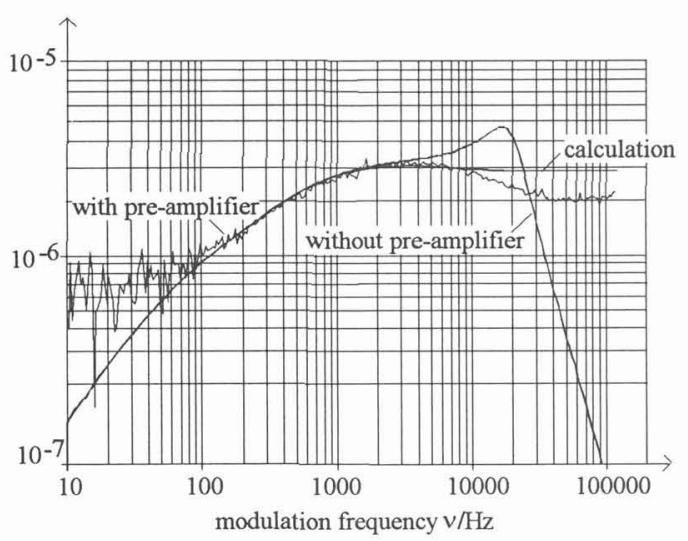

Fig.2. Amplitude of the PM-playback signal from a homogeneously magnetized $\mathrm{CrO}_{2}$-tape as a function of the modulation frequency. Experimental data measured with and without preamplifier and theoretical curve obtained with relation given above.

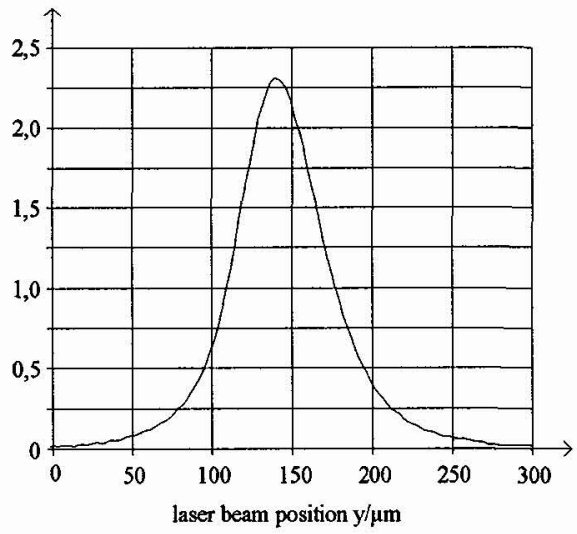

Fig.3. Lateral variation of the PM-playback amplitude of a homogeneously magnetized tape across the gap of the recorder head.

Experiments were performed on magnetic tapes of $\mathrm{CrO}_{2}, \mathrm{Fe}_{2} \mathrm{O}_{3}$ and ferrochrome. The tapes had been either homogeneously magnetized parallel to the tape direction or a $50 \mathrm{~Hz}$ track had been recorded on the tapes by a recorder. Both, laterally resolved measurements at a fixed modulation frequency and depth dependent measurements with the laser focused at the centre of gap area have been performed. All signals had been normalised with respect to an unmagnetized tape. The commercial recorder head showed a bandpass characteristic with a roll-off frequency of about $12 \mathrm{kHz}$ (Fig.2). Inserting a home-made preamplifier the signals could be detected up to $100 \mathrm{kHz}$. A most peculiar property of the PM-playback signal is the increase of the amplitude by nearly two orders of magnitude between $10 \mathrm{~Hz}$ and $10 \mathrm{kHz}$. This particular frequency dependence results from the competition of the law of induction and the thermal wave behaviour. In the same frequency range the phase of the PM-playback signal decreases by about $90^{\circ}$. Similar behaviours are observed for the other tape materials. The measured frequency dependence of both the amplitude and phase angle are in fairly good agreement with the behaviour calculated on the basis of the equation given above. The lateral resolution ability of the PM-playback is demonstrated in Fig.3. Scanning the intensity laser spot along the direction of the tape the amplitude of the PM playback signal provides a measure of the lateral sensitivity of the recorder head. As $\mathrm{M}$ is constant for the homogeneously magnetized tape, the trace in Fig. 3 essentially reproduces the variation of the magnetic fringing-field across the head gap at the position of the tape. In addition, the lateral variation of a sinusoidal track on a tape has been resolved by displacing the tape underneath the laser spot.

The most original and most promising result in view of future applications of the PM-playback technique is the depth dependent observation of a $50 \mathrm{~Hz}$ tack on a chromium oxide tape. During the writing process the sinusoidally varying input voltage produces a correlated amplitude variation of the Karlquist field. As a consequence the tape gets magnetized only in those regions perpendicular to the tape surface where the magnetizing field of the head gap remains above the coercitive field of the hysteresis loop. Therefore, for a sinusoidal track also a sinusoidal variation of the magnetization depth along the tape is expected. For the first time, this depth variation could be measured nondestructively, applying the frequency dependent PMplayback technique. In order to visualise the lateral variation, the laser was always focused on the centre of the gap, whereas the tape was shifted step by step underneath the laser in the tape direction. 


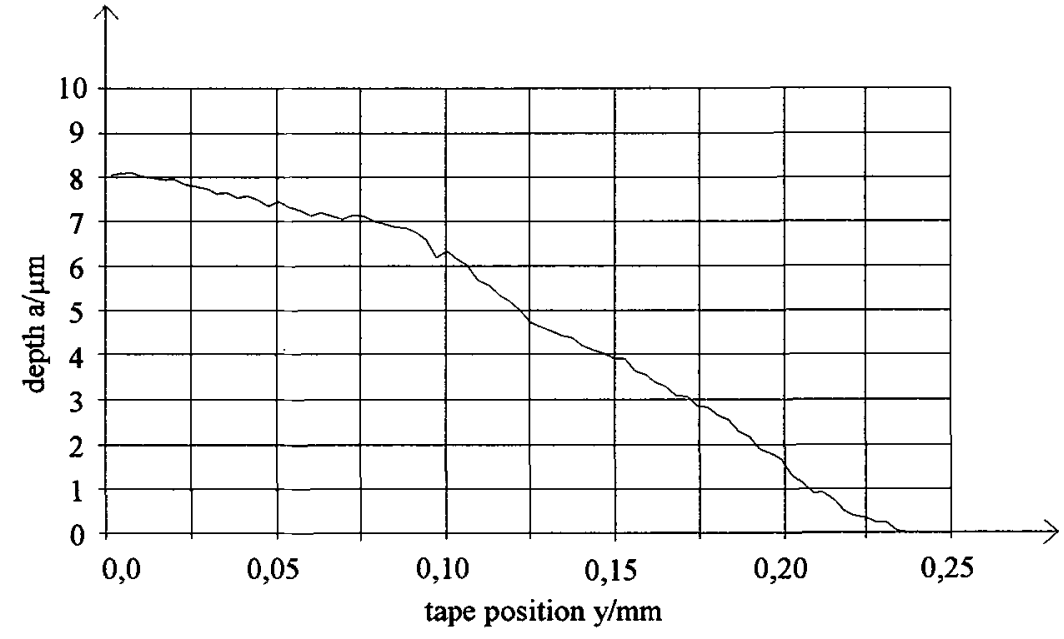

Fig.4. Depth dependence of the magnetized along a sinusoidal track recorded on a $\mathrm{CrO}_{2}$ tape. Average of measurements at 10 different frequencies in the range $300 \mathrm{~Hz}$ to $100 \mathrm{kHz}$.

The amplitude traces as a function of the modulation frequency yield clear evidence of the influence of the depth variation particularly in the high frequency range.

The data have been analysed using equation (1). In a first step the magnetisation profile across the tape had been approximated by a steplike function where $M$ is zero above a critical distance a. The adjustment could be improved considerably in a second step by assuming smooth decrease of $\mathrm{M}$ around $\mathbf{a}$. The descent of $\mathrm{M}$ from $80 \%$ to $20 \%$ of its saturation value is
characterised by a decay distance $b$. Using $\mathbf{a}$ and $\mathbf{b}$ as adjustable parameters, the measured data could be consistently analysed. Fig. 4 shows the result obtained for the mean magnetization depth a. The decay distance deduced from this measurement was $\mathbf{b}=5 \mu \mathrm{m}$.

\section{CONCLUSION}

Photothermally modulated playback relies on the reading of magnetically recorded signals by a thermal wave induced voltage in a recorder head. This novel technique offers both a lateral as well as a depth resolution of the magnetization traces recorded parallel to the moving direction of the tape. Magnetisation components aligned perpendicular to this direction do not contribute to the PM-playback signal. The depth discrimination is most effective at high modulation frequencies above the audio range. The results of this measurement for the first time reveal nondestructively the depth profile of a sinusoidal signal trace. The PM-playback measurements were also able to provide detailed information on the descent of the magnetisation distribution.

This work is supported by the Deutsche Forschungsgemeinschaft, Kenmwort "Photothermik".

\section{REFERENCES}

[1.] Pelzl J. and Netzelmann U., Topics in Current Physics 47, (ed. P. Hess, Springer Verlag, Berlin 1989) p.331.

[2.] Orth Th., Netzelmann U. and Pelzl J., Appl. Phys. Lett. 53 (1988) pp. 1979-1981.

[3.] Pelzl J., Netzelmann U., Orth Th. and Kordecki R., Photoacoustic and Photothermal Phenomena II, (eds. J.C. Murphy et al., Springer Series in Optical Sciences, Springer Verlag Berlin 1990) Vol. 62, p.2.

[4.] Karlquist O., Trans. Roy. Inst. Techn. Stockholm 86, (1954).

[5.] Kaack M., Master thesis, (Ruhr-Universität Bochum, 1993) pp.24-36. 